\title{
Biomechanical Analysis of Reducing Sacroiliac Joint Shear Load by Optimization of Pelvic Muscle and Ligament Forces
}

\author{
J. J. M. Pel, C. W. Spoor, A. L. Pool-Goudzwatrd, G. A. Hoek van Dijke, and C. J. Snijders \\ Department of Biomedical Physics and Technology, Erasmus MC, PO Box 2040, Rotterdam 3000 CA, The Netherlands
}

(Received 5 April 2007; accepted 18 September 2007; published online 18 January 2008)

\begin{abstract}
Effective stabilization of the sacroiliac joints (SIJ) is essential, since spinal loading is transferred via the SIJ to the coxal bones, and further to the legs. We performed a biomechanical analysis of SIJ stability in terms of reduced SIJ shear force in standing posture using a validated static 3-D simulation model. This model contained 100 muscle elements, 8 ligaments, and 8 joints in trunk, pelvis, and upper legs. Initially, the model was set up to minimize the maximum muscle stress. In this situation, the trunk load was mainly balanced between the coxal bones by vertical SIJ shear force. An imposed reduction of the vertical SIJ shear by $20 \%$ resulted in $70 \%$ increase of SIJ compression force due to activation of hip flexors and counteracting hip extensors. Another 20\% reduction of the vertical SIJ shear force resulted in further increase of SIJ compression force by $400 \%$, due to activation of the transversely oriented $\mathrm{M}$. transversus abdominis and pelvic floor muscles. The $\mathbf{M}$. transversus abdominis crosses the SIJ and clamps the sacrum between the coxal bones. Moreover, the pelvic floor muscles oppose lateral movement of the coxal bones, which stabilizes the position of the sacrum between the coxal bones (the pelvic arc). Our results suggest that training of the $\mathbf{M}$. transversus abdominis and the pelvic floor muscles could help to relieve SI-joint related pelvic pain.
\end{abstract}

Keywords-Static forces, Sacroiliac joints, Pelvis, Pelvic floor muscles, Human posture.

\section{INTRODUCTION}

The human body uses an ingenious 3-D framework of bones, joints, muscles, and ligaments for posture and movement. In upright posture, the trunk load passes the sacroiliac joints (SIJ). The orientation of the SIJ surfaces, however, is more or less in line with the direction of loading, which induces high shear forces between sacrum and coxal bones. ${ }^{34}$ The SIJ have a strong passive, viscoelastic ligamentous system for

Address correspondence to J. J. M. Pel, Department of Biomedical Physics and Technology, Erasmus MC, PO Box 2040, Rotterdam 3000 CA, The Netherlands. Electronic mail: j.pel@ erasmusmc.nl providing stability. These ligaments are vulnerable for creep during constant trunk load and need to be protected against high SIJ shear forces. ${ }^{19}$ From a biomechanical point of view, an active muscle corset that increases the compression force between the coxal bones and the sacrum could protect the ligamentous system and support the transfer of trunk load to the legs and vice versa. Interlocking of the SIJ may be promoted by transversely oriented muscles, e.g., M. transversus abdominis, M. piriformis, M. gluteus maximus, $\mathrm{M}$. obliquus externus abdominis, and $\mathbf{M}$. obliquus internus abdominis, which has been described as self-bracing. ${ }^{33-35}$ However, due to the complex lines of action of (counteracting) muscles and ligaments in the pelvic region, it is difficult to demonstrate the contribution of transversely oriented muscles to SIJ stability, in vitro as well as in vivo.

In the past, a number of biomechanical models of the lumbosacral region (spine and pelvis) have been developed to study the aetiology of low back pain (LBP) in relation to (over)loading of the lumbar spine ${ }^{1,9,11,36}$ and the pelvis. ${ }^{10,27}$ Most of these models dealt with mechanical stability in terms of muscle $3,4,18$ and compression forces between (lumbar) vertebrae. ${ }^{2,8,12,20,22,28}$ A different approach is to relate LBP to overloading of SIJ and nearby ligaments, for example the iliolumbar ligaments. ${ }^{24,30}$ The load transfer through the SIJ was studied using a static, 3-D biomechanical simulation model based on the musculoskeletal anatomy of the trunk, pelvis, and upper legs. ${ }^{15}$ This simulation model calculates forces in muscles, ligaments, and joints that are needed to counterbalance trunk weight and other external forces. It was shown that this simulation model underestimated antagonistic muscle activity, but a good agreement was found for agonist muscle activity. The number of passive structures in the model was small, for example no joint capsules were incorporated. Therefore, the model was only valid for postures in which none of the joints were near an end position. 
The aim of the present study was to determine which muscles have to become active in the $3-\mathrm{D}$ pelvic simulation model when there is an imposed reduction of the vertical SIJ shear force.

\section{MATERIALS AND METHODS}

\section{The 3-D Simulation Model}

The present study was performed using the validated, 3-D simulation model as described by Hoek van Dijke et al. ${ }^{15}$ The model is based on the musculoskeletal anatomy of the trunk, pelvis, and upper legs, including muscle and ligament attachment sites, crosssectional areas of muscles and the direction of muscle, ligament, and joint reaction forces. The geometry of this model was based on structures extracted from MRI slices and from previously published data on lumbar spine ${ }^{8}$ and upper leg ${ }^{4,17}$ geometry. Figure 1a illustrates, in frontal and median view, the bones on which muscle and ligament forces act in the simulation model. These are the lowest thoracic vertebra, five lumbar vertebrae, the sacrum, the left and right coxal bones and the left and right femurs. The vertebrae are treated as a single structure. The arrangement of the bones depends on the static posture for which muscle forces are calculated, for example standing with or without trunk flexion. A description of the model equilibrium, its optimization scheme and validation of some of the parameters is presented in the Appendix. In the present study, we focus on the compression and shear forces in the SIJ. These forces are represented as perpendicular vectors. The normal vector of the SIJ surface has an oblique direction ( $x y z=0.365$, $\pm 0.924,0.114)$. Compression force is defined along this normal vector and can only vary in magnitude. One of the two components of the SIJ shear force was defined in the $Y Z$-plane $(x y z=0, \pm 0.123,0.992)$. This force is denoted as the vertical SIJ shear force. Directions of the SIJ compression force and the SIJ vertical shear force in the $Y Z$-plane are shown in Fig. 1a, left panel. Figure $1 \mathrm{~b}$ shows the vectors representing the most important muscle and ligament forces in the pelvic region superimposed on the bones. Bone shapes are for illustration purpose only; they are not part of the simulation model. In total, the model contains 100 vectors for muscle forces, 8 vectors for ligament forces, and 22 vectors for joint forces; see Table 1 for a list of all the structures.

\section{Simulations and Data Analyses}

A first simulation, with the model in standing posture and a trunk weight of $500 \mathrm{~N}$, showed that the vertical shear SIJ force was $563 \mathrm{~N}$ on each side of the

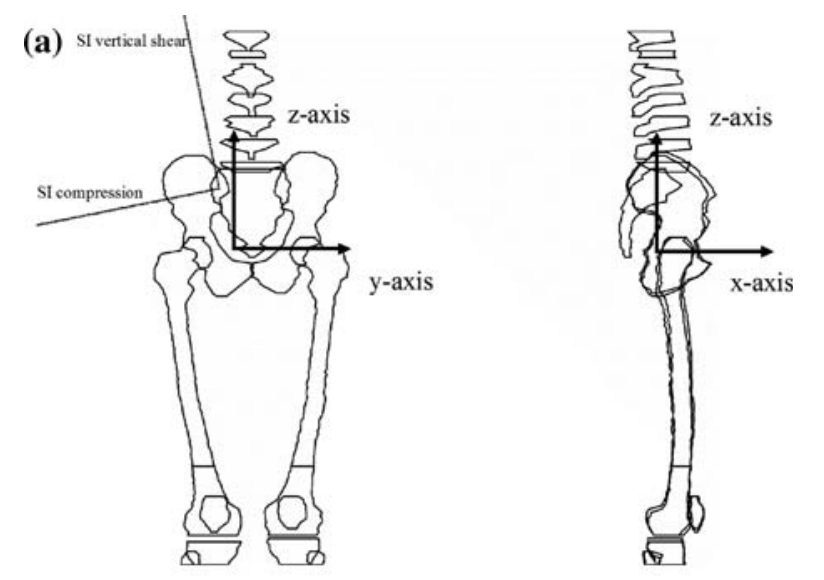

(b)

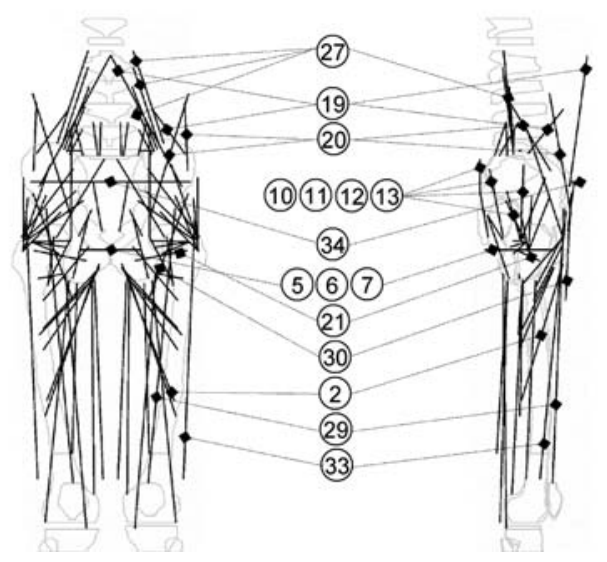

FIGURE 1. Panel (a) shows the bones on which the muscles, ligaments and joint reaction forces act in the frontal plane (left) and the median plane (right): the lowest thoracic vertebra, five lumbar vertebrae, the sacrum, the left and right coxal bones, and the left and right femurs. The coordinate system is defined with the origin halfway between the rotation centers of the hip joints. Axes: $x$ posterior, $y$ left, $z$ vertical. Panel (b) shows, superimposed on the bones, the vectors representing the most important force components in the frontal plane (left) and the median plane (right), see also Table 1. The labels in this panel refer to a selection of the muscle structures listed in Table 1.

sacrum. To find the muscles that promote sacroiliac joint stability, the maximum value for the vertical SIJ shear force was decreased in steps of $30 \mathrm{~N}(\sim 5 \%$ of the initial vertical SIJ shear force). Theoretically, lowering of the imposed vertical SIJ shear force to $0 \mathrm{~N}$ could induce a non-physiological equilibrium between muscle, ligament, and joint forces. In addition, when the model was set in $30^{\circ}$ flexion, the force in the iliolumbar, the sacrotuberal, and the posterior sacroiliac ligaments was $250 \mathrm{~N}$. This value was set as a maximum physiological ligament force in the simulation model in the upright position to prevent overloading of the pelvic ligaments that were implemented in the model. The following criteria were defined to warrant a physiological solution for muscle and ligament forces. 
TABLE 1. List of muscles, ligaments, and joints and the number of vectors describing the forces used in the simulation model on transferring trunk load from lumbar spine via the pelvis to the upper legs (unilateral), see also Fig. 1.

\begin{tabular}{|c|c|c|c|}
\hline & Name of structure & Number of elements & Remark \\
\hline 1 & M. adductor brevis & 2 & Upper and lower muscle \\
\hline 2 & M. adductor longus & 1 & \\
\hline 3 & M. adductor magnus & 3 & Upper, middle and lower muscle \\
\hline 4 & M. biceps femoris & 1 & \\
\hline 5 & M. coccygeus & 1 & Pelvic floor muscle \\
\hline 6 & M. iliococcygeus & 1 & Pelvic floor muscle \\
\hline 7 & M. pubococcygeus & 1 & Pelvic floor muscle \\
\hline 8 & M. gemellus inferior & 1 & \\
\hline 9 & M. gemellus superior & 1 & \\
\hline 10 & M. gluteus maximus & 2 & Femur-sacrum and ilium muscle \\
\hline 11 & M. gluteus maximus facia & 2 & Ilium-femur and trunk muscle \\
\hline 12 & M. gluteus medius & 3 & Upper, middle, and lower muscle \\
\hline 13 & M. gluteus minimus & 3 & Upper, middle, and lower muscle \\
\hline 14 & M. gracilis & 1 & \\
\hline 15 & M. iliacus & 1 & \\
\hline 16 & M. longissimus & 1 & \\
\hline 17 & M. iliocostalis & 1 & \\
\hline 18 & M. multifidus & 1 & \\
\hline 19 & M. obliquus externus abdominis & 2 & Ventral and dorsal muscle \\
\hline 20 & M. obliquus internus abdominis & 2 & Ventral and dorsal muscle \\
\hline 21 & M. obturatorius externus & 1 & \\
\hline 22 & M. obturatorius internus & 1 & \\
\hline 23 & M. pectineus & 1 & \\
\hline 24 & M. piriformis & 1 & \\
\hline 25 & M. psoas & 2 & Upper and lower muscle \\
\hline 26 & M. quadratus femoris & 1 & \\
\hline 27 & M. quadratus lumborum & 5 & Sacrum—rib12, L1, L2, L3 and L4 muscle \\
\hline 28 & M. rectus abdominis & 1 & \\
\hline 29 & M. rectus femoris & 1 & \\
\hline 30 & M. sartorius & 1 & \\
\hline 31 & M. semimembranosus & 1 & \\
\hline 32 & M. semitendinosus & 1 & \\
\hline 33 & M. tensor fasciae latae & 1 & \\
\hline 34 & M. transversus abdominis & 1 & \\
\hline A & Iliolumbar ligament & 1 & Transversal plane \\
\hline B & Posterior sacroiliac ligament & 1 & Transversal plane \\
\hline $\mathrm{C}$ & Sacrospinal ligament & 1 & \\
\hline $\mathrm{D}$ & Sacrotuberous ligament & 1 & \\
\hline I & L5-S1 joint & 3 & Shear (two directions) and compression \\
\hline II & SI joint & 3 & Shear (two directions) and compression \\
\hline III & Hip joint & 3 & Shear (two directions) and compression \\
\hline IV & Knee joint & 3 & Shear (two directions) and compression \\
\hline $\mathrm{V}$ & Pubic symphysis & 1 & Compression \\
\hline
\end{tabular}

1. Muscle tension must not exceed $240 \mathrm{kPa}^{16}$;

2. Lowering of the maximum vertical SIJ shear force must result in reduction of the total SIJ shear force (combination of vertical and horizontal shear);

3. Ligament force must not exceed $250 \mathrm{~N}$.

A muscle was included for further analysis when it produced at least $15 \%$ of the maximum muscle stress during the simulation. For all muscles, the maximum muscle stress depended on the calculated minimum muscle stress (see optimization criterion 1 in the Appendix). Two muscle groups were analyzed separately: (1) the muscles that increased at least $80 \%$ in force after the first simulation step and (2) the muscles that increased at least 10 times in force after completion of the simulation series.

\section{RESULTS}

Table 2 summarizes the muscle (de)activation pattern when the maximum vertical SIJ shear force was stepwise decreased. Initially, vertical SIJ shear force was $563 \mathrm{~N}$ (on each side of the sacrum) at a trunk load of $500 \mathrm{~N}$. The angle between the normal direction of the SIJ surface and the direction of the total SIJ 
TABLE 2. Summary of the structures that stabilize the sacroiliac joints in terms of lowered shear.

\begin{tabular}{|c|c|c|c|c|c|c|c|c|c|}
\hline \multirow{2}{*}{$\begin{array}{l}\text { Reduction of sacroiliac shear } \\
\text { SIJ (vertical shear) }\end{array}$} & \multirow{2}{*}{$\begin{array}{r}\text { Initial } \\
563\end{array}$} & \multicolumn{8}{|c|}{ Preset value $(N)$} \\
\hline & & 533 & 503 & 473 & 443 & 413 & 383 & 353 & 323 \\
\hline Structures & & & & & Force $(\Lambda$ & & & & \\
\hline M. adductor longus & 9 & 18 & 18 & 18 & 18 & 9 & & & \\
\hline M. coccygeus & & 1 & 1 & 1 & 1 & 2 & 4 & 10 & 20 \\
\hline M. iliococcygeus & & 1 & 1 & 1 & 1 & 2 & 4 & 10 & 20 \\
\hline M. pubococcygeus & & & & & & & 1 & 2 & 6 \\
\hline M. gluteus medius (lower) & 7 & 11 & 13 & 14 & 15 & 27 & 44 & 30 & \\
\hline M. gluteus medius (middle) & 5 & 8 & 11 & 10 & 10 & 10 & 29 & 41 & \\
\hline M. gluteus medius (upper) & & & & & & & & 42 & 90 \\
\hline M. gluteus minimus (lower) & & 3 & 3 & 3 & 3 & 4 & 7 & & \\
\hline M. gluteus minimus (middle) & 5 & 10 & 11 & 11 & 11 & 5 & 10 & 12 & \\
\hline M. gluteus minimus (upper) & 8 & 17 & 18 & 18 & 17 & 5 & 11 & 16 & 25 \\
\hline M. iliacus & 47 & 47 & 50 & 54 & 58 & 68 & 88 & 85 & 102 \\
\hline M. obliquus externus abdominis & 21 & 18 & 17 & 14 & 12 & & & & \\
\hline M. obliquus internus abdominis & 15 & 20 & 20 & 23 & 27 & 34 & 29 & & \\
\hline M. obturatorius externus & 15 & 4 & 4 & 4 & 5 & 8 & & & \\
\hline M. pectineus & 8 & 18 & 18 & 18 & 18 & 6 & & & \\
\hline M. piriformis & 18 & 26 & 28 & 25 & 22 & & & & \\
\hline M. psoas (lower) & 64 & 50 & 46 & 27 & & & & & \\
\hline M. rectus abdominis & 27 & 27 & 29 & 31 & 34 & 37 & 51 & 76 & 83 \\
\hline M. rectus femoris & 34 & 34 & 36 & 39 & 42 & 49 & 64 & 62 & 50 \\
\hline M. sartorius & & 16 & 17 & 18 & 19 & 16 & 22 & 14 & \\
\hline M. tensor fasciae latae & 31 & 31 & 33 & 35 & 38 & 44 & 58 & 85 & 79 \\
\hline M. transversus abdominis & 3 & 5 & 5 & 6 & 7 & 21 & 32 & 53 & 82 \\
\hline Iliolumbar ligament & & & & & & & 53 & 250 & 250 \\
\hline Posterior sacroiliac ligament & & 26 & 49 & 62 & 73 & 147 & 250 & 250 & 250 \\
\hline Sacrospinal ligament & & 38 & 150 & 159 & 147 & 145 & 132 & 106 & 74 \\
\hline Sacrotuberous ligament & 206 & 151 & 21 & & & & & & \\
\hline SIJ (compression) & 92 & 121 & 130 & 142 & 154 & 229 & 473 & 607 & 633 \\
\hline SIJ (horizontal shear) & -132 & -141 & -160 & -154 & -142 & -154 & -208 & -226 & -233 \\
\hline Total SIJ shear & 579 & 551 & 528 & 497 & 465 & 441 & 436 & 419 & 398 \\
\hline Angle of SIJ reaction force $\left({ }^{\circ}\right)$ & 81 & 78 & 76 & 74 & 72 & 63 & 43 & 35 & 32 \\
\hline Maximum muscle tension (kPa) & 37 & 37 & 39 & 42 & 45 & 53 & 69 & 125 & 247 \\
\hline
\end{tabular}

Included are those muscles that produced at least $15 \%$ of the maximum muscle stress after each simulation.

The muscles printed italic increased at least $80 \%$ in force after the first simulation step. The muscles printed bold increased at least 10 times in force after completion of the simulation series.

reaction force was $81^{\circ}$, indicating that mainly vertical shear force acted through the SIJ, see Fig. 2a. Force equilibrium was mainly achieved by activation of $\mathrm{M}$. abdominal oblique (internus and externus), M. iliacus, M. psoas, M. rectus abdominis, M. rectus femoris, $M$. tensor fasciae latae, and loading of the sacrotuberous ligament.

When the maximum vertical SIJ shear force was decreased from 563 to $443 \mathrm{~N}$ in steps of $30 \mathrm{~N}$, the SIJ compression force increased by about $70 \%$. Force equilibrium was obtained, amongst others, by activation of some of the muscles with a hip flexion component (M. adductor longus, M. iliacus, M. pectineus, and $\mathrm{M}$. sartorius, $\mathrm{M}$. rectus femoris) and some of the counteracting hip extensors (MM. gluteus medius and minimus and $\mathrm{M}$. piriformis). Most of these muscles became (more) active after we lowered the maximum vertical SIJ shear force by $30 \mathrm{~N}$. This led to unloading of the sacrotuberous ligaments and loading of the sacrospinal ligaments. The angle between the normal direction of the SIJ surface and the direction of the total SIJ reaction force was reduced to $72^{\circ}$, indicating that a combination of reduced vertical SIJ shear and increased SIJ compression could balance the trunk load on the sacrum, see Fig. $2 b$.

Further stepwise reduction of the vertical SIJ shear force resulted in a sharp rise of the maximum muscle stress. The simulation series ended with exceeding the maximum physiological muscle stress when the vertical SIJ shear force was decreased to about $60 \%$ of its initial value. Surprisingly, activation of some of the hip flexors and extensors had decreased or even disappeared. This was not the case for the MM. gluteus medius and minimus. In this simulation, force equilibrium was obtained by activation of the transversely oriented M. transversus abdominis (ventral to the SIJ) 


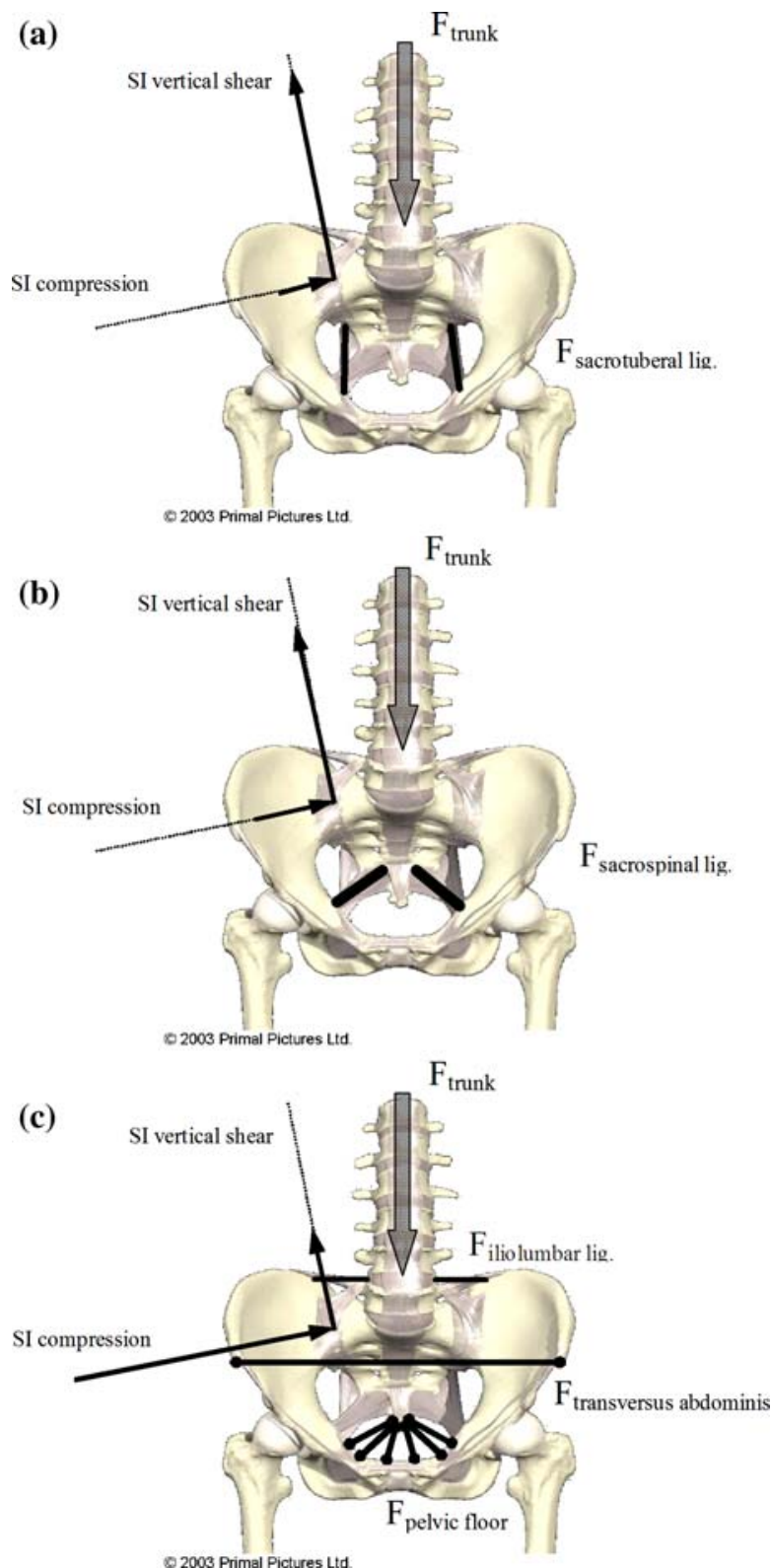

FIGURE 2. Directions of the force in the frontal plane exerted by the right ilium through the SIJ on the sacrum as a reaction to trunk load, $F_{\text {trunk }}$. Panel (a): initial loading condition without limitation of the vertical shear component $(563 \mathrm{~N}$, see under "initial" in Table 2). This condition led to loading of the sacro-

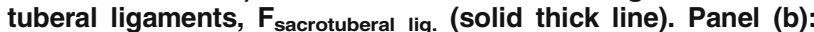
loading condition with the vertical shear component preset at a $120 \mathrm{~N}$ lower level than the initial value (see under $443 \mathrm{~N}$ in Table 2). This condition led to loading of the sacrospinal liga-

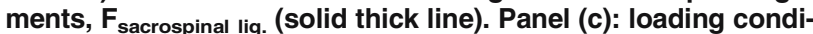
tion with the vertical shear component preset at a $240 \mathrm{~N}$ lower level than the initial value (see under $323 \mathrm{~N}$ in Table 2). In this situation, SIJ compression force increased by $\sim 400 \%$, mainly by M. transversus abdominis, $F_{\text {transversus abdominis, and the pelvic }}$ floor, $F_{\text {pelvic floor }}$, muscle forces. The location of these muscles is schematically drawn by the thick solid lines, including the M. pubococcygeus, the M. iliococcygeus and the M. coccygeus (as drawn from the mid to the lateral position). It also led to loading of the iliolumbar ligaments to the maximum allowed force $F_{\text {iliolumbar lig. of }} 250 \mathrm{~N}$, (solid thick line). 3-D images copyright of Primal Pictures Ltd. http://www.primalpictures.com. and the pelvic floor muscles, i.e., the M. coccygeus, the M. iliococcygeus, and the M. pubococcygeus (caudal to the SIJ). This resulted in further reduction of the angle between the normal direction of the SIJ surface and the direction of the total SIJ reaction force to $35^{\circ}$, see Fig. 2c. This indicates that the SIJ compression force, which increased by about $400 \%$ and the reduced vertical SIJ shear force, now clamped the sacrum between the coxal bones, see Fig. 2c. The MM. gluteus medius and minimus contributed to some extent to this increased compression due to a distinct force component in the transverse direction. To maintain force equilibrium, increased SIJ compression led to loading of the iliolumbar and posterior sacroiliac ligaments to the preset maximum value of $250 \mathrm{~N}$.

\section{DISCUSSION}

In the present study, the simulation model predicted muscle and ligament forces in the pelvic region when there was an imposed reduction of the vertical SIJ shear force. Initially, the forces acting through the SIJ were mainly vertical shear forces, see Fig. 2a. These forces were not only caused by trunk load, but also by muscles that acted in the longitudinal direction of the spine, for example the M. psoas and M. rectus abdominis. As a result of the forward bending moment, the sacrotuberous ligament was loaded. This large ligament protects the SIJ against excessive flexion of the sacrum relative to the coxal bones. The controlled reduction of the vertical SIJ shear force with $30 \mathrm{~N}$ forced some muscles that act as hip flexors and hip extensors to become active. Due to their transverse orientation, especially the MM. gluteus medius and minimus and $M$. piriformis contributed to the increased compression force between the coxal bones and the sacrum. However, these muscles did not contribute enough to self-bracing of the SIJ, because the total force through the SIJ still mainly acted in vertical direction. When the vertical SIJ shear was further reduced to about $60 \%$ of its initial value, the simulation model predicted that self-bracing mainly resulted from the transverse muscles ventrally (M. transversus abdominis) and caudally (pelvic floor) to the SIJ. In this situation, some of the hip flexors and extensors reduced in activity, for example the M. piriformis. Although the M. piriformis has a transverse orientation and crosses the SIJ, its contribution was minimized by the simulation program because this muscle also induces vertical SIJ shear force. The pelvic floor muscles, the M. coccygeus and M. pubo-, and iliococcygeus, contribute to the stabilization with respect to the sacrum. It has been suggested that this stabilization by force closure has an analogy with a classical 


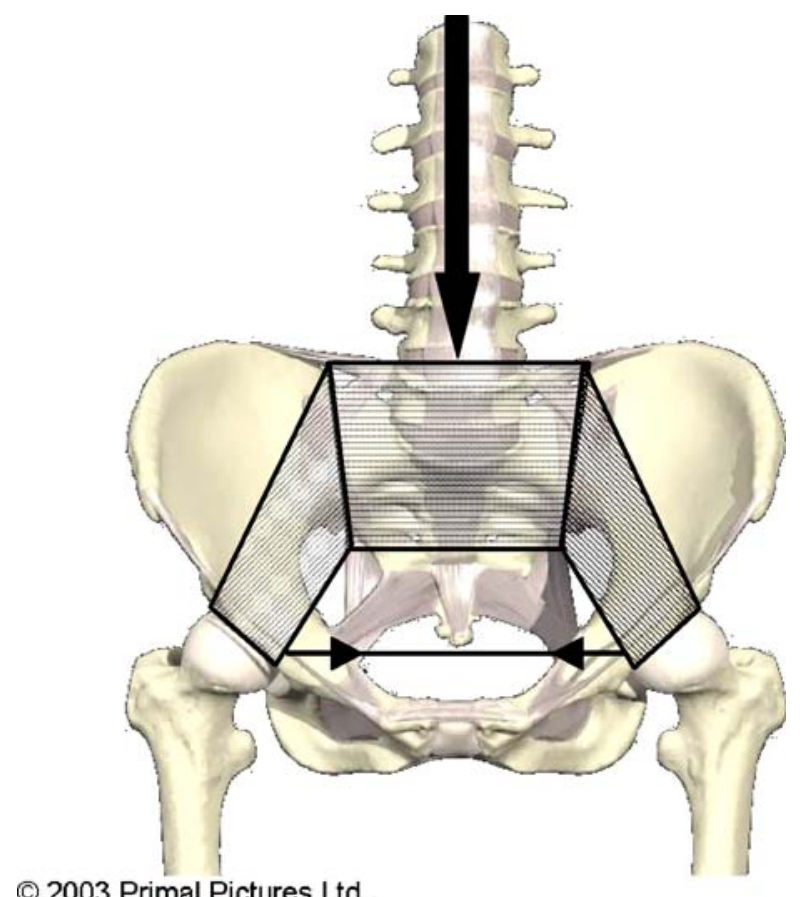

(C) 2003 Primal Pictures Ltd.

FIGURE 3. Analogy of pelvic bones supporting the trunk with a classical stone arc. The $M$. transversus abdominis and the pelvic floor muscles caudal to the SIJ mainly oppose lateral movement of the coxal bones. Spinal loading is transferred mainly by compression forces through the SIJ to the coxal bones and further down to the legs. 3-D images copyright of Primal Pictures Ltd. http://www.primalpictures.com.

stone arc. ${ }^{33}$ When sideways displacement of both ends of the arc is opposed, mechanical equilibrium of the stones is achieved by compression forces and not by shear forces. In the pelvis, the pelvic floor muscles may help the coxal bones to support the sacrum by compression forces, while shear forces between sacrum and coxal bones are minimized, see Fig. 3. Note that the SI compression force is defined as the force acting perpendicular to the SIJ surface. Therefore, decreasing or increasing this force will not alter the shear forces. The articular surfaces of the SIJ are irregular which results in bony interdigitation in the SI joint space. SIJ shear force calculated in the simulation model thus reflects the combination of real joint friction and friction due to this intermingling of bones. The real joint friction forces may be extremely small considering the extremely low coefficients of friction between the articular surfaces. The majority of the shear force is effectuated as normal contact pressures due to the bony interdigitation in the SI joint space. It was not possible to calculate the percentage of shear in terms of joint friction force. This requires a more detailed description of the SIJ surfaces.

The simulation model predicts that simultaneous contraction of the $\mathrm{M}$. transversus abdominis and pelvic floor muscles, i.e., the M. coccygeus, the M. iliococcygeus, and the $M$. pubococcygeus, contribute to lowering of the vertical SIJ shear forces, increasing of the SIJ compression and hence increasing of the SIJ stability. We emphasize that this simulation model was set up to estimate the forces acting in the pelvic region under static conditions and that the outcome of the simulations must be interpreted with caution. ${ }^{5}$ Nevertheless, in a previous study co-contraction was shown of pelvic muscles and M. transversus abdominis. ${ }^{26}$ This result and the prediction of our simulation model suggest that a protective mechanism against high SIJ shear forces may exist in humans. This mechanism has been investigated in vivo and in vitro. The contribution of the M. transversus abdominis to SIJ stability was shown in an in vivo study in patients with LBP. ${ }^{25} \mathrm{An}$ in vitro study in embalmed human pelvises showed that simulated pelvic floor tension increased the stiffness of the pelvic ring in female pelvises. ${ }^{23}$ It is worthwhile to further investigate the contribution of both muscle groups simultaneously, not only during stiffness measurements of the SIJ but also during lumbo-pelvic stability tests based on increased intra-abdominal pressure (IAP). It was shown that the pelvic floor muscles, in combination with abdominal muscles and the diaphragm, may control and/or sustain IAP to increase lumbar spine stability as well. ${ }^{7,14}$

In the present study, the ligament forces were not allowed to exceed $250 \mathrm{~N}$. The distribution between muscle and ligament forces depended on the maximum muscle stress as formulated in the first optimization scheme as presented in the Appendix. Increasing the maximum ligament forces might result in a lower maximum muscle stress, which could lead to a different muscle activation pattern to stabilize the SIJ. A small sensitivity test, however, showed that when the ligament forces were allowed to exceed the $250 \mathrm{~N}$ up to $500 \mathrm{~N}$ and in a next step up to $750 \mathrm{~N}$, the model calculated a similar muscle activation pattern. The outcome of the present study also depended on the choice of optimization criteria and the magnitude of the crosssectional areas of the muscles. The influence of different criteria was previously investigated for muscle forces in the leg. ${ }^{21}$ Indeed, various choices led to different calculated forces, but the obtained solutions were qualitatively similar, as was the case in our model. When we developed the model, other optimization criteria were also tested, for example minimization of the sum of muscle forces. However, minimization of the sum of squared muscle stresses yielded the most plausible solutions. The model cannot account for anatomical variations or detailed variation in muscle attachment sites. Obviously, direct comparison between the model predictions and the outcome of in vivo force measurements in the SIJ are not available, 
so there is no data to confirm the outcome of the present study. Nevertheless, EMG recordings of (superficial) abdominal and back muscles in various postures showed higher M. abdominal oblique internus activity when standing upright than resting on one leg and tilting the pelvic backwards. ${ }^{33}$ This muscle is considered as one of the self-bracing muscles of the SIJ. It was hypothesized that when standing on one leg, the shear load on the contralateral SIJ is diminished. Posterior tilt of the pelvis with less lumbar lordosis may than lead to less M. psoas major muscle load on the spine meaning less shear load on the SIJ. These findings indirectly support our findings that transversely oriented muscles reduce SIJ shear forces. We emphasize that the present model served as a tool to investigate the general relations between muscle and ligament forces in the pelvic region. The present simulations results may lead to the development of a new SIJ stabilizing training-program to reduce pain induced by high SIJ shear forces. The effectiveness of such a program, however, can only be tested with an intervention study.

The simulation model predicted unloading of the sacrotuberous and loading of the iliolumbar and posterior sacroiliac ligaments when the vertical SIJ shear was forced to reduce. This loading of the dorsal ligaments resulted from the absence of transversely oriented muscles at the dorsal side of the SIJ to counterbalance activation of the $\mathrm{M}$. transverse abdominis at the ventral side of the SIJ. Loading of the iliolumbar ligament has been related to LBP. ${ }^{24}$ It was shown that in sitting position, the stepwise backward movement of an erect trunk (from upright position into a slouch) resulted in forward flexion of the spine combined with backward tilt of the sacrum relative to the pelvis. ${ }^{32}$ It was shown, that this movement into a sudden or sustained slouch might cause loading of the well-innervated iliolumbar ligaments near failure load. ${ }^{31}$ The co-contraction that exists between the deep abdominal M. transversus abdominis and the deep back extensor $M$. multifidus presumably retains lumbo-pelvic stability. ${ }^{13}$ In the future, we intend to extend the model with co-contraction between the M. transversus abdominis, the M. multifidus, and the pelvic floor muscles to study prevention of (over)loading of pelvic ligaments at different static postures.

\section{CONCLUSIONS}

Effective stabilization of the SIJ is essential in transferring spinal load via the SIJ to the coxal bones and the legs. A biomechanical analysis of the upright standing posture showed that activation of transversely oriented abdominal $\mathrm{M}$. transversus abdominis and pelvic floor, i.e., M. coccygeus and M. pubo- and iliococcygeus muscles would be an effective strategy to reduce vertical SIJ shear force and thus to increase SIJ stability. The force equilibrium in this situation induced loading of the iliolumbar and posterior sacroiliac ligaments. The $\mathrm{M}$. transversus abdominis crosses the SIJ and clamps the sacrum between the coxal bones. Moreover, the pelvic floor muscles oppose lateral movement of the coxal bones, which stabilizes the position of the sacrum (the pelvic arc).

\section{APPENDIX}

This Appendix summarizes the model equilibrium, its optimization scheme and model validation and reliability. Part of the text is a short summary of previously published data. ${ }^{15}$ A detailed description of the model will be provided on request.

\section{Biomechanical Model Equilibrium}

Figure 1a illustrates, in frontal and median view, the bones on which muscle and ligament forces act in the simulation model. The origin of its coordinate system is located between the rotation centers of the hip joints. The ligament and joint forces are the result of counteracting the external loads and the muscles forces. All forces act along straight lines, and in principle, each muscle (see also Table 1) or ligament has one line of action, see Fig. 1b. The simulation model quantifies the muscle, ligament, and joint forces to warrant force equilibrium of the complete model. In the present study, the trunk load is the only external force applied. It is also possible to include an external reaction force as a backing support. The trunk load transfers from the lumbar spine, via the pelvis to the lower legs on the basis of the prescribed posture. The static equilibrium equations for the trunk, the coxal bones, and the sacrum are based on a free-body diagram of each part. All forces and moments exerted on each part, including the weight, the external loads, the muscle, and ligament forces, and the joint reaction forces, have to be in equilibrium. Reaction forces in joints balance the other forces. The trunk has a reaction force in the L5-S1 joint, the sacrum has additional reaction forces in the SIJ, and the two coxal bones have reaction forces in the SIJ and the hip joints. In the present study we focus on the vertical SIJ shear forces. These are the net sum of trunk load, muscle, and ligament forces, and reaction forces acting in vertical direction on the vertebrae, the sacrum, and the coxal bones. Reaction forces are calculated in this order, that is starting with the trunk and ending with the ground reaction force (which is 
not given as output of the model). Leaving out the ground reaction forces on the feet does not mean that the model floats: these forces are just not needed in the equilibrium equations of the trunk, the pelvis, and the femurs.

\section{Force Vector Optimization Scheme}

The pelvis was represented as three separate links (left and right coxal bones and sacrum). The left and right femurs and lowest thoracic vertebra were included as links as well. Muscles and ligaments were modeled as vector forces, and muscle, ligament, and joint reaction forces between the separate links balanced the loading of the pelvis. Several sources were combined to complete the anatomical data set. A set of 164 transversal MRI-scans of a healthy young male adult enabled the quantification of the 3-D coordinates of muscle and ligament attachments sites and the magnitude of cross-sectional areas to calculate muscle stresses. A computer program was written to describe the positions of all attachment sites in different postures. For each load situation, the equilibrium of the links had to be calculated. Since the model is statically indeterminate, additional criteria were formulated to come to unique solution of muscle, ligament, and joint reaction forces. The program GAMS $^{6}$ was used to determine the optimum solution for the forces. It calculated the magnitudes of 130 force vectors based on a preset load (e.g., the trunk load) using first a linear and second a quadratic optimization criterion. Since the present study focused on static postures, the first linear criterion was as follows:

- The maximum muscle stress was minimized. The upper limit for muscle stress valid for all muscles was minimized, which was considered as maximizing the endurance time. This criterion only minimized muscle stresses, not the ligament forces. The ligament and joint reaction forces were just part of the optimum solution for the muscle forces.

This criterion did not necessarily lead to a unique solution. For example: in case a trunk muscle, e.g., the M. erector spinae determined the lowest possible value for the maximum muscle stress, still an infinite number of solutions may exist for balancing muscle forces around the hip. Thus, a supplementary, quadratic criterion was used:

- The sum of squared muscle stresses was minimized, with the additional constraint that no muscle stress was allowed to exceed the value that followed from the first criterion. This led to distribution of the load over synergic muscles within the boundary of the first criterion. However, strictly upholding this additional constraint led to unrealistic activity of muscles when a small decrease of the maximum muscle stress was achieved by recruiting many muscles. In addition, this second criterion could enhance the increase of ligament forces, since the constraint was only applied to muscle stresses. To prevent that ligament forces became too high, the maximum muscle stress was allowed to exceed the calculated lowest maximum muscle stress from the first criterion by $10 \%$. This increase of the maximum stress was allowed to reduce the recruitment of inefficient muscles, but it also tempered excessive loading of ligament forces.

Figure 4 shows a flow chart of optimizing muscle and ligament forces and stresses in terms of position, loading, forces, and stresses.

\section{Model Validation and Reliability}

The presented biomechanical model enabled the analysis of load transfer from the upper body to the pelvis and legs. The number of passive structures in

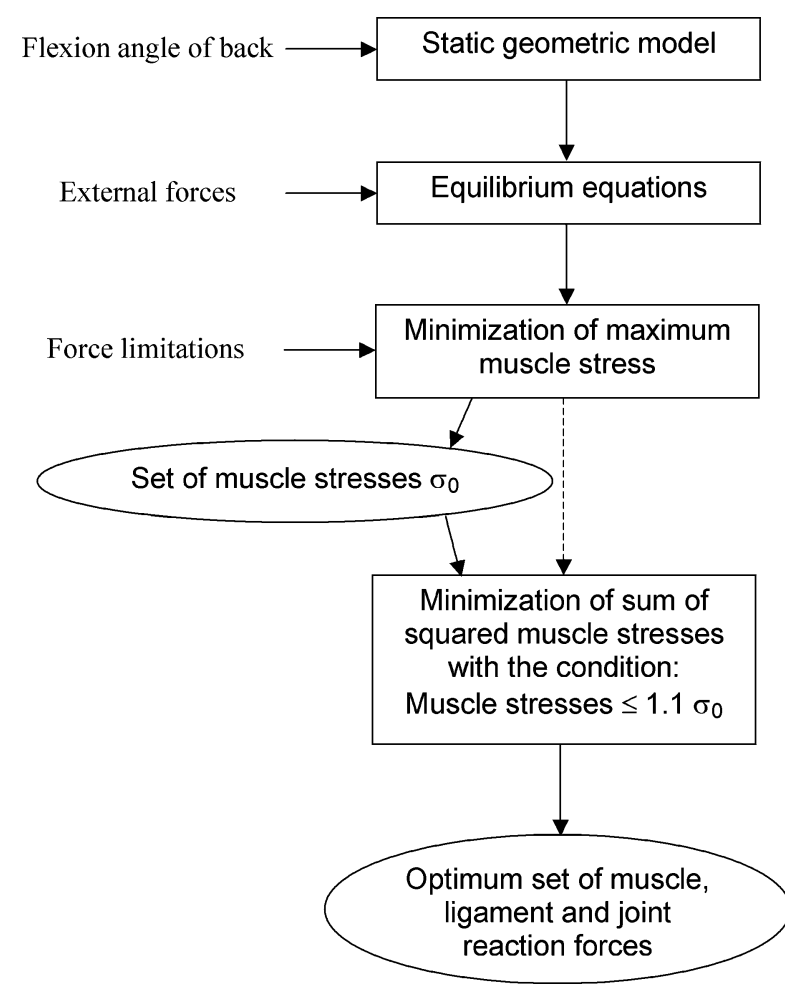

FIGURE 4. A flow chart of the optimization scheme used to calculate the optimum set of muscle, ligament and joint reaction forces on the basis of geometry, positions, external forces, and force limitations. 
the model is small, for instance no joint capsules were incorporated into the model; it was only valid for postures that were not close to the ends of the range of motion of the joints. All forces acted along straight lines, and in principle, each muscle or ligament had one line of action, see Fig. 1b. However, muscles with broad attachment sites were either defined by a number of lines of action in parallel, e.g., the M. gluteus maximus and the $\mathrm{M}$. obliquus externus abdominis or in series, e.g., the M. psoas major, see Ref. 15 for a detailed description. For example: the M. gluteus maximus had attachments to the sacrum (partially via the sacrotuberous ligament), the coxal bone and the thoracolumbar fascia. Since forces in these three parts had fundamentally different effects on the load in the SIJ, this muscle was modeled as three separate force vectors. The three lines of action that represented the M. gluteus maximus, were given a relative crosssectional area according to the mass proportion of these muscle parts. ${ }^{15}$

The reliability of the model was tested by comparing calculated muscle stresses with measured EMG activities described in the literature. The experiments in the papers that were selected had to be accurately described and EMG activity of various muscles in at least one load situation or EMG activity of a single muscle in a variety of load situations had to be reported. This resulted in model simulations of (1) forward hipflexion with the trunk held straight, ${ }^{3}(2)$ an isometric axial trunk torque exercise, ${ }^{18}$ and (3) various asymmetric sagittal and frontal plane loading conditions. ${ }^{29}$ On average, a good correlation between muscle EMG activity and calculated muscle stresses was found, ranging from 0.869 to 0.984 . For example, the normalized EMG values (using a scaling factor $1 \%$ of the maximum voluntary contraction of $0.0037 \mathrm{~N} / \mathrm{mm}^{2}$ ) and calculated muscle stresses at different hip flexion angles showed good proportionality (correlation coefficient of 0.904). This was not the case for the activity of the M. quadratus lumborum (slightly higher EMG value) and the M. erector spinae (slightly lower EMG value). Overall, a good agreement was found for agonist muscle activity, but antagonistic muscle activity was underestimated. The aim of the present model was the development of a tool to investigate the general relations between muscle and ligament forces in the pelvic region, rather than to focus on the contribution of individual muscles. To enable the use of EMG data as input in the model, a facility was made to define upper and lower limits for each muscle force vector. It also provides the possibility to simulate co-contraction of antagonistic muscles in the pelvic region. A similar facility was made for ligament and joint reaction forces. In the present study, the ligament forces were not allowed to exceed a maximum of $250 \mathrm{~N}$.

\section{ACKNOWLEDGMENT}

This research was supported by the Anna Foundation.

\section{REFERENCES}

${ }^{1}$ Adams, M. A., and P. Dolan. Recent advances in lumbar spinal mechanics and their clinical significance. Clin. Biomech. 10:3-19, 1995

${ }^{2}$ Anderson, C. K., D. B. Chaffin, G. D. Herrin, and L. S. Matthews. A biomechanical model of the lumbosacral joint during lifting activities. J. Biomech. 18:571-584, 1985. ${ }^{3}$ Andersson, E. A., L. I. E. Oddsson, H. Grundstrom, J. Nilsson, and A. Thorstensson. EMG activities of the quadratus lumborum and erector spinae muscles during flexion-relaxation and other motor tasks. Clin. Biomech. 11:392-400, 1996.

${ }^{4}$ Brand, R. A., R. D. Crowninshield, C. E. Wittstock, D. R. Pedersen, C. R. Clark, and F. M. van Krieken. A model of lower extremity muscular anatomy. J. Biomech. 104:304 310, 1982.

${ }^{5}$ Brand, R. A., D. R. Pedersen, and J. A. Friederich. The sensitivity of muscle force predictions to changes in physiologic cross-sectional area. J. Biomech. 19:589-596, 1986.

${ }^{6}$ Brooke, A., D. Kendrick, and A. Meeraus. GAMS - A User's Giude. Washington, DC: GAMS Development Corporation, 1996.

${ }^{7}$ Cholewicki, J., K. Juluru, and S. M. McGill. Intraabdominal pressure mechanism for stabilizing the lumbar spine. J. Biomech. 32:13-17, 1999.

${ }^{8}$ Cholewicki, J., and S. M. McGill. Mechanical stability of the in vivo lumbar spine: implications for injury and chronic low back pain. Clin. Biomech. 11:1-15, 1996.

${ }^{9}$ Dolan, P., and M. A. Adams. Recent advances in lumbar spinal mechanics and their significance for modelling. Clin. Biomech 16:S8-S16, 2001.

${ }^{10}$ Goel, V. K., and N. L. Svensson. Forces on the pelvis. J. Biomech. 10:195-200, 1977.

${ }^{11}$ Gracovetsky, S., H. F. Farfan, and C. A. Lamy. Mathematical model of the lumbar spine using an optimized system to control muscles and ligaments. Orthop. Clin. N. Am. 8:135-153, 1977.

${ }^{12}$ Granata, K. P., and W. S. Marras. An EMG-assisted model of loads on the lumbar spine during asymmetric trunk extensions. J. Biomech. 26:1429-1438, 1993.

${ }^{13}$ Hides, J. A., G. A. Jull, and C. A. Richardson. Long-term effects of specific stabilizing exercises for first-episode low back pain. Spine 26:E243-E248, 2001.

${ }^{14}$ Hodges, P. W., J. E. Butler, D. K. McKenzie, and S. C. Gandevia. Contraction of the human diaphragm during rapid postural adjustments. J. Physiol. 505:539-548, 1997.

${ }^{15}$ Hoek van Dijke, G. A., C. J. Snijders, R. Stoeckart, and H. J. Stam. A biomechanical model on muscle forces in the transfer of spinal load to the pelvis and legs. J. Biomech. 32:927-933, 1999

${ }^{16}$ Jaspers, R. T., H. Degens, and P. A. Huijing. Specific tension of intact and skinned muscle fibres. J. Biomech. 39:S56, 2006.

${ }^{17}$ Koopman, B. The three-dimensional analysis and prediction of human walking. PhD thesis, Twente Unversity, The Netherlands, 1989. 
${ }^{18}$ McGill, S. M. Electromyographic activity of the abdominal and low back musculature during the generation of isometric and dynamic axial trunk torque: implications for lumbar mechanics. J. Orthop. Res. 9:91-103, 1991.

${ }^{19}$ McGill, S. M., and S. Brown. Creep response of the lumbar spine to prolonged full flexion. Clin. Biomech. 7:1975-1983, 1992.

${ }^{20}$ McGill, S. M., N. Patt, and R. W. Norman. Measurement of the trunk musculature of active males using CT scan radiography: implications for force, moment generating capacity about the L4/L5 joint. J. Biomech. 21:329-341, 1988.

${ }^{21}$ Pederson, D. R., R. A. Brand, C. Cheng, and J. S. Arora. Direct comparison of muscle force predictions using linear and nonlinear programming. J. Biomech. 10:128-136, 1987.

${ }^{22}$ Plamondon, A., M. Gagnon, and D. Gravel. Moments at the L5/S1 joint during asymmetrical lifting: effect of different load trajectories and initial load positions. Clin. Biomech. 10:128-136, 1995.

${ }^{23}$ Pool-Goudzwaard, A. L., G. A. Hoek van Dijke, M. van Gurp, P. Mulder, C. J. Snijders, and R. Stoeckart. Contribution of pelvic floor muscles to stiffness of the pelvic ring. Clin. Biomech. 19:564-571, 2004.

${ }^{24}$ Pool-Goudzwaard, A. L., G. J. Kleinrensink, C. J. Snijders, C. Entius, and R. Stoeckart. The sacroiliac part of the iliolumbar ligament. J. Anat. 199:457-463, 2001.

${ }^{25}$ Richardson, C. A., C. J. Snijders, J. A. Hides, L. Damen, M. S. Pas, and J. Storm. The relationship between the transversus abdominis muscle, sacroiliac joint mechanics and low back pain. Spine 27:399-405, 2002.

${ }^{26}$ Sapsford, R. R., and P. W. Hodges. Contraction of the pelvic floor muscles during abdominal manoeuvre. Arch. Phys. Med. Rehab. 82:1081-1088, 2001.

${ }^{27}$ Scholten, P. J., A. B. Schultz, C. W. Luchies, and J. A. Miller. Motions and loads within the human pelvis: a biomechanical model study. J. Orthop. Res. 6:840-850, 1988.
${ }^{28}$ Schultz, A. B., and G. B. Andersson. Analysis of loads on the lumbar spine. Spine 6:76-82, 1981.

${ }^{29}$ Seroussi, R. E., and M. H. Pope. The relationship between trunk muscle electromyography and lifting moments in the sagittal and frontal planes. J. Biomech. 20:135-146, 1987.

${ }^{30}$ Snijders, C. J. Standing, sitting and lying. In: Basic Biomechanics of the Musculoskeletal System, edited by M. Nordin and V. H. Frankel. New York: Lippincott Williams \& Wilkins, 2001, pp. 420-436.

${ }^{31}$ Snijders C. J., P. F. Hermans, R. Niesing, G. J. Kleinrensink, and A. Pool-Goudzwaard. Effects of slouching and muscle contraction on the strain of the iliolumbar ligament. Man. Ther. Epub ahead of print, 2007.

${ }^{32}$ Snijders, C. J., P. F. G. Hermans, R. Niesing, C. W. Spoor, and R. Stoeckart. The influence of slouching and lumbar support on iliolumbar ligaments, intervertrebal discs and sacroiliac joints. Clin. Biomech. 19:323-329, 2004.

${ }^{33}$ Snijders, C. J., M. T. L. M. Ribbers, H. de Bakker, R. Stoeckart, and H. Stam. EMG recordings of abdominal and back muscles in various standing postures: validation of a biomechanical model on sacroiliac joint stability. $J$. Electromyogr. Kines. 8:205-214, 1998.

${ }^{34}$ Snijders, C. J., A. Vleeming, and R. Stoeckart. Transfer of lumbosacral load to iliac bones and legs. Part 1: biomechanics of self-bracing of the sacroiliac joints and its significance for treatment and exercise. Clin. Biomech. 8:285294, 1993.

${ }^{35}$ Snijders, C. J., A. Vleeming, and R. Stoeckart. Transfer of lumbosacral load to iliac bones and legs. Part 2: loading of the sacroiliac joints when lifting in a stooped posture. Clin. Biomech. 8:295-301, 1993.

${ }^{36}$ Tracy, M. F. Three-dimensional force model of the lowback for simple computer programming. Clin. Biomech. 5:175-179, 1990 . 